\title{
Implicaciones valorativas sobre el rol de la investigación científica en el redimensionamiento de la Educación Superior.
}

\author{
Valuation implications on the role of scientific research in the resizing of \\ Higher Education.
}

Sandro Oscar Muela Proaño. ${ }^{1}$, Luis Ricardo Villacis Monar. ${ }^{2}$, María Fernanda Duran Veloz, ${ }^{3} \&$ Oscar Paúl Tanqueño Colcha. ${ }^{4}$

Recibido: 15-12-2020 / Revisado: 05-01-2021 /Aceptado: 12-01-2021/ Publicado: 05-02-2021

\begin{abstract}
:
DOI: https://doi.org/10.33262/concienciadigital.v4i1.1.1558

Introduction: the knowledge society imposes on the universities the need for a paradigm shift in the training of professionals, in order to bring career profiles closer to the occupational needs in the different sectors of the administration of productive operations and services, where the use of information and communication technologies, transform the dynamics of laws and essential relationships between objects and phenomena of reality. Objective: to offer timely reflections that allow to revalue the role of scientific research in the context of higher education, as well as the approach of a set of evaluative guidelines and intrinsic qualities, which allow the reader from their area of performance to redefine the role of the research you do at your university. Methodology: it is based on methods of theoretical, empirical level. Results: Scientific Research is a conscious process, determining essential laws and relationships of the research object that are independent of man's consciousness (although they are specified through it), in order to solve a scientific problem. Conclusion the traditional notion of scientific research and its procedure in the contemporary university, understanding it evolves according to the human need for new knowledge.
\end{abstract}

Key words: scientific research, professional training, higher education.

\section{Resumen}

Introducción: la sociedad del conocimiento impone a las universidades la necesidad de un

\footnotetext{
${ }^{1}$ Universidad Estatal de Bolívar, Facultad de Ciencias Administrativas, Gestión Empresarial e Informática, Guaranda, Ecuador, smuela@ueb.edu.ec, https://orcid.org/0000-0002-3357-0806

${ }^{2}$ Universidad Estatal de Bolívar, Facultad de Ciencias Administrativas, Gestión Empresarial e Informática, Guaranda, Ecuador, lvillacis@ueb.edu.ec, https://orcid.org/0000-0001-7932-366X.

${ }^{3}$ Universidad Estatal de Bolívar, Facultad de Ciencias Administrativas, Gestión Empresarial e Informática, Guaranda, Ecuador, mduran@ueb.edu.ec, https://orcid.org/0000-0001-8992-6210

${ }^{4}$ Universidad Estatal de Bolívar, Facultad de Ciencias Administrativas, Gestión Empresarial e Informática, Guaranda, Ecuador, otanqueno@ueb.edu.ec, https://orcid.org/0000-0002-5718-335X
} 
cambio de paradigmas en la formación de los profesionales, en función de acercar los perfiles de las carreras, a las necesidades ocupacionales en los diferentes sectores de la administración de operaciones productivas y de servicios, donde el empleo de las tecnologías de la información y las comunicaciones, transforman la dinámica de las leyes y relaciones esenciales entre los objetos y fenómenos de la realidad. Objetivo: ofrecer reflexiones oportunas que permitan revalorizar el papel de la investigación científica en el contexto de la educación superior, así como el abordaje de un conjunto de pautas valorativas y cualidades intrínsecas, que le permitan al lector desde su área de desempeño redefinir el rol de la investigación que realiza en su universidad. Metodología: se fundamenta, en métodos de nivel teórico, empírico. Resultados: la Investigación Científica es un proceso consciente, determinándose leyes y relaciones esenciales del objeto de investigación que son independiente de la conciencia del hombre (aunque se concretan a través del mismo), en aras de resolver un problema científico. Conclusión la noción tradicional de investigación científica y su proceder en la universidad contemporánea entendiéndose evoluciona en función de la necesidad el ser humano de nuevo conocimiento.

Palabras clave: Investigación científica, formación de profesionales, educación

\section{Introducción:}

En la educación superior existen una gran diversidad de criterios acerca de los enfoque de la investigación y el papel que ejerce está en los procesos de formación de profesionales, pero también en este sentido muchos autores piensan y coinciden en el criterio a cerca de la necesidad de un cambio sustancial en la educación, tal como la conocemos actualmente, pero ese cambio, para que sea sostenible, debe estar fundamentado en evidencias y partir de una realidad objetiva y compartida por la comunidad generadora del proceso formativo.

Por esas razones resulta prudente dirigir el propósito del presente artículo, a reflexionar en torno al papel de la investigación científica educacional en el contexto de la educación superior, en aras de encontrar a través de este análisis reflexivo las alianzas conducentes desde diversos puntos de vista, para trazar un camino basado en las múltiples alternativas que permite la toma de decisiones en la investigación; como respuesta a la reducción de las brechas entre la formación y el empleo, sustentada en la aplicación del método científico en función de la transformación de los modelos educativos vigentes, y acercar la enseñanza a las competencias que requiere la nueva dinámica organizacional mundial basada en la aplicación globalizada de las tecnologías de información y las comunicaciones (TIC).

En este sentido, se esclarecerán a modo de opinión autoral, sobre la base de la sistematización de diversas fuentes sobre el tema, algunas de las pautas que esbozan la significatividad de la metodología de la investigación científica en la educación superior, rasgos elementales sobre lo que se debe sustentar cualquier innovación docente y la propia investigación educativa, si se pretende reducir las brechas entre la formación y el empleo, como uno de los principales desafíos del siglo XXI.

\section{Metodología:}


El desarrollo de la presente investigación se fundamenta, en métodos de nivel teórico, empírico, como: análisis y síntesis, cualitativo, histórico-lógico, lo cual implica una profunda revisión bibliográfica para abstraer el fundamento teórico a la realidad actual.

\section{Resultados:}

Las reflexiones sobre la educación para el siglo XXI, se atribuyen, entre otros autores, a la Organización de las Naciones Unidas para la Educación, la Ciencia y la Cultura, por sus siglas en inglés UNESCO, que prescribe a la educación como un instrumento indispensable para el progreso de la humanidad en camino hacia la paz, la libertad y la justicia social; con una función esencial que es el desarrollo continuo de la persona y la sociedad (UNESCO, 2017).

La educación superior en el siglo XXI se presenta con una fisonomía de cambio, que conlleva características como: la masificación en la matriculación, el aprendizaje a distancia, la educación para toda la vida, la tensión entre lo mundial y lo local, el dilema de la mundialización de la cultura, las nuevas tecnologías de la información, la concentración en los problemas inmediatos, la competencia y la igualdad de oportunidades, el desarrollo del conocimiento y la capacidad de asimilación del ser humano, el respeto al pluralismo, la superación del sí mismo y la incertidumbre valorativa (UNESCO, 2016).

Del mismo modo, la educación superior se enfrenta a desafíos y dificultades relativos al financiamiento, a la igualdad de condiciones en el acceso a los estudios y permanencia en ellos, mejor perfil del docente, la formación basada en competencias, mejora y calidad en la enseñanza, investigación y consultoría, pertinencia de los programas de estudio, la internacionalización de la universidad, el uso de la tecnología como parte de la metodología didáctica en aula, y demás características que responden al cambio como una constante de momento (Cid, Cuadra, Cuevas, \& Villalobos, 2017).

En este escenario, la docencia, como actividad básica fundamental, que es la actividad asociada al conocimiento y aprendizaje, demanda del profesor incitar el interés por aprender, cómo aprender y mantener al día estos conocimientos; para alcanzar estas obligaciones se necesita contar con capacidades, conocimientos, habilidades, actitudes y valores (Díaz, 2017).

El papel del docente como reproductor de conocimiento ha sido reemplazado por un orientador de los aprendizajes, de este modo, son los estudiantes quienes deben saber buscar, procesar y utilizar el nuevo contenido aprendido, en función del conocimiento previo. Esto demanda una concepción del proceso de aprendizaje que provea la adquisición de las capacidades, identifique estrategias comunes para solucionar problemas y desarrolle capacidades socio-afectivas como valores, actitudes, motivaciones y emociones. El rol del profesor no se ve limitado a la adquisición de conocimientos y al desarrollo de destrezas, sino que también tiene una gran importancia el desarrollo de los valores (Mas-Torelló \& OlmosRueda, 2016).

Otra actividad básica del docente, es la investigación, que ha sido objeto de cambios como 
parte de las transformaciones académica y formativas necesarias a implementar por las universidades en los últimos años, reformas que han dado como resultado una estrecha combinación entre la enseñanza y la investigación; hoy se fomenta la investigación competitiva, la creación de equipos multidisciplinarios formados por miembros de universidades de diversos países, donde el desarrollo de investigación debe estar asociado a un proceso de planificación sostenible y que responde a líneas y grupos de investigación que demuestren un impacto en la sociedad (Armijos, Barreno, \& Sánchez, 2015).

Para producir conocimiento científico hay que producir científicos, por lo que queda la inquietud acerca de la capacidad de dudar en las generaciones de alumnos que se recibe en el aula. El marco normativo supone a un alumno universitario con potencial científico que dejó de ser un sujeto pasivo del aprendizaje y ha pasado a ser un sujeto activo capaz de aumentar el conocimiento o refutar el disponible. El docente que trabaja con este alumno debe estar apto para llevar a cabo el desarrollo de la investigación y la adquisición del perfil profesional que la universidad busca formar (Espinosa \& Aguilar, 2017).

El cambio en la capacidad investigativa de la universidad se debe en buena parte a la inclusión de la tecnología y acceso a las tecnologías de la información y comunicación, que facilita el acceso a bibliotecas virtuales, bases de datos, documentos y demás fuentes de información; la capacidad de manejo y procesamiento de bases de datos, la comunicación al momento del conocimiento científico, el intercambio de conocimiento con otros investigadores en todo el mundo a través de correo, videoconferencia, mensajería; la capacidad de almacenamiento de gran cantidad de información en medios portátiles o en entornos virtuales denominados la nube y el interés de organizaciones por proponer y financiar la investigación en temáticas específicas (Zambrano, 2017).

Pensar en la investigación desde corrientes educacionales sobre el conocimiento de la ciencia, la cultura, lo humano y su epistemología, enmarcan una acción que señalan la objetividad, y que permiten una construcción de formas pedagógicas que ayudan en generar formas de poder en la colectividad, que a la postre servirán como bases sólidas para ayudar a las distintas instituciones en consolidar un proyecto educativo en pertinencia a investigación, como una forma de control y la gestación de las desigualdades hoy en día existentes, y en estos tiempos, nuevas formas de acumulación y dominación (Ortega, 2009; Ramírez, Chacón y Valencia, 2018).

En la esfera de la educación, así como en algunos grupos subalternos en el mundo desarrollado y en diferentes actores críticos de otros continentes, se ha concretado, una concepción de trabajo educativo basado en la investigación, que se caracteriza por ser una acción política, la cual busca transformar las condiciones de control, dominio y formas de sujeción de los actores, las comunidades e instituciones. Para lograrlo busca generar la conciencia crítica y las dinámicas sociales que impulsen que los grupos construyan sus propias formas de asociación y organización que los convierte en sujetos colectivos, constructores de su historia (Gadotti, 2003). 
Así, las organizaciones, señalan Mendoza, López, \& Salas (2016), "son entes que generan sus propios objetivos y diseñan la forma de cómo alcanzarlos teniendo en cuenta, necesariamente, las influencias del entorno social" (p. 62). En este sentido las universidades no están exentas de esta funcionalidad, y es de allí que, en las organizaciones de la educación superior, cualquiera que sea su ámbito, se hace preciso introducir una estratégica como respuesta social (Mendoza, López y Salas, 2016)

De tal manera, la investigación como estrategia debe ser concebida similar al pensamiento estratégico que según Arellano (2004) citado por Mendoza y López (2015), "hace referencia a la forma del pensamiento que se dirige hacia la creatividad, imaginación, y búsqueda de opciones innovadoras y realistas, como potenciación de lo posible enmarcado dentro de un proyecto de construcción intencional de la realidad" (p. 154).

En este entendido, exponen Hernández y Jiménez (2015), que estas redes "no reclama sólo la ética individual de las personas que componen las organizaciones, sino que ven un ente con responsabilidad propia económica, social y medioambiental a la que debe exigírsele comportamientos acordes a la misma" (p. 11). Según su razón de ser mediante las múltiples manifestaciones del poder en nuestra colectividad, esto con la intención en construir un espíritu de emancipación humana, para no desarrollar procesos de poder que controlan ni dominan, ni permitir que otros lo realicen en su propia vida (Torres, 2007).

Con lo cual "se podrá avanzar en el conocimiento sobre las condiciones de creación y de permanencia... de manera más amplia, del dinamismo del sistema social”. (Maculan, Jiménez y Castellanos, 2015, p. 16). Va a ser el ejercicio de reconocer los contextos como lugares de saber. Por ello, la investigación busca devolver al acto educativo: diferencia, heterogeneidad y multiplicidad fundadas en una diversidad cultural, social y cognitiva

En el marco reflexivo en torno a estas cualidades, es importante considerar que la investigación es definida como un conjunto de procesos sistemáticos y críticos que se aplican al estudio de un fenómeno, también puede ser definida como "una serie de métodos para resolver problemas cuyas soluciones necesitan ser obtenidas a través de una serie de operaciones lógicas, tomando como punto de partida datos objetivos" (Arias, s.f).

Con esta definición se manifiesta que el Método Científico que utilizan en ciencias exactas u otros métodos en ciencias sociales tienen la función de apoyar la resolución de problemas a partir de datos específicos, teniendo que cumplir los criterios de pertinencia y relevancia. Se debe tener en cuenta que las investigaciones se originan por ideas, primer acercamiento a la realidad objetiva (desde la perspectiva cuantitativa), subjetiva (desde la perspectiva cualitativa) o intersubjetiva (la óptica mixta), que habrá de investigarse (Porlan, 1987).

Los cambios de posturas en la investigación científica educacional, están asociados a una profunda transformación, que implicó nuevas formas de organización social, económica y política; a partir de esta nueva configuración social, se empezó a difundir y aceptar desde finales del siglo pasado, una visión basada en la idea central de que el conocimiento y la 
información se convertirían en la clave para la generación y distribución del poder en la sociedad, sustituyendo así a los recursos naturales y al dinero (Tedesco, 1998); en este marco de referencia surgió la sociedad del conocimiento, hecho que implicó una transformación profunda en las formas de vida y de relación con nuevos conceptos de tiempo y espacio (Torres, y Campos, 2016).

Varios países han trascendido la sociedad de la información para dar paso a la sociedad del conocimiento que, desde una perspectiva humanista y compleja, se enfoca en resolver problemas con base en la colaboración de las tecnologías de la información y la comunicación (TIC), y de los valores universales como el respeto, honestidad y la equidad (Tobón, 2017; Silva 2016).

De acuerdo con la sociedad del conocimiento, la educación desempeña un papel muy importante en el desarrollo de nuevas competencias en los ciudadanos, preparándole para enfrentar los retos sociales en la actualidad; uno de los objetivos principales de la educación, es formar ciudadanos calificados que respeten los derechos humanos, sean responsables ante la sociedad y garanticen un proceso educativo eficaz (Caliskan, Kuz, \& Kuzu, 2017). Por su parte, Buckworth (2017) plantea que hoy en día la preparación de los docentes se define en torno a las expectativas académicas y al éxito pedagógico demostrable en las aulas; esto implica dotar al estudiante de las competencias profesionales que le permitan desarrollar con éxito su práctica docente.

Lo anterior indica que, para formar un ciudadano para la sociedad del conocimiento, el profesor debe desarrollar competencias docentes necesarias para que, a su vez, las propicie en sus alumnos; esto se convierte en un verdadero desafío para los docentes de educación superior: implica vencer varios obstáculos, entre ellos, el diseño y operación de una reforma educativa con un antiguo paradigma que no presenta cambios sustanciales y de profundidad, operando con diseños y programas curriculares desactualizados y lejanos a las necesidades de aprendizaje de los estudiantes. En tanto que el modo de enseñar sea tradicional, modular, por proyectos, etc., pero no toque la estructura curricular del plan y programa de cada nivel, la realidad educativa se mantendrá sin cambios (Canto, 2016).

Los modelos de formación docente en educación superior podrían ser reformados de acuerdo con los nuevos planteamientos de la sociedad del conocimiento, siempre y cuando consideren imprescindibles el cambio y la innovación en los programas formativos, ligados a la integración de conocimientos y los involucramientos de los actores institucionales; junto con ello, se busca que se muestre la utilidad de los conocimientos producidos, para que sean utilizados en la resolución de problemas del contexto (Padierna, 2016); en el plano de la formación docente, se le solicita al maestro que innove su práctica y que transforme los procesos de aprendizaje en sus alumnos, pero desafortunadamente no se le dan los elementos necesarios para ello.

Imbernón (2016) plantea que cuando un profesional se encuentra ante un contexto social, laboral y educativo, necesariamente requiere de una formación específica en varias 
capacidades y una situación laboral que permita desarrollar bien su trabajo. En el caso de los pedagogos, requieren formarse para la sociedad del conocimiento, lo cual implica la apropiación crítica y selectiva de la información, significa saber qué se quiere y cómo aprovechar el conocimiento en la solución de los problemas de contexto; así, la formación profesional debe estar plenamente identificada en este proceso (Martínez, 2014).

Para lograr una verdadera transformación social y educativa, se requiere de congruencia en las expectativas sociales y de educación; la principal función de esta es desarrollar y ofrecer herramientas para el aprendizaje de nuevas formas de comunicación y búsqueda de la información, mismas que constituyan el instrumento principal para desenvolverse en el mundo: si el contexto y las formas de aprender cambian, resulta lógico pensar que la educación debe experimentar modificaciones (Pozuelo, 2014).

En la transformación los programas y modelos de formación, los docentes tienen que convertirse en aprendices al identificar debilidades y al proponer estrategias para la superación de las dificultades que se le presenten en su práctica profesional (Moriya, Schlünzen, Nascimento, 2016); por lo tanto, la formación docente se convierte en un factor esencial en la preparación del maestro, al que tiene que habilitar para enfrentar nuevas fórmulas de trabajo y, en consecuencia, nuevas tareas (Olmedo, y Farrerons, 2014). Estas nuevas tareas implican una modificación de sus formas de aprender y de enseñar, incluyendo la utilización de nuevas herramientas para el aprendizaje; con ello también se convierte en la figura central para la incorporación de las TIC en los procesos de formación de sus estudiantes (Martínez, 2013).

El incluir las TIC es una pieza clave en la transformación y en los procesos de enseñanzaaprendizaje para lograr una calidad en la educación (Cabero, y Marin, 2014). En el contexto de este cambio paradigmático, se ha erigido el perfil y las competencias profesionales docentes como referentes para la transformación pedagógica y educativa, conllevando automáticamente a nuevos planteamientos en el diseño, desarrollo y evaluación de los procesos formativos (Tejada, y Ruiz, 2016). Ante esta perspectiva, el docente debe ser innovador y capaz de transformar su propio proceso de formación en un espacio de indagación, que utilice estrategias y recursos apropiados para la promoción de aprendizajes significativos en sus futuros alumnos (Moreno, 2015).

Dicha situación ha originado varias iniciativas como la estrategia para la enseñanza y la formación técnica de la Organización de las Naciones Unidas para la Educación, la Ciencia y la Cultura (UNESCO, por sus siglas en inglés), que ha promovido en su agenda 2030 el desarrollo sostenible; para ello ha prestado especial atención al desarrollo de competencias técnicas y profesionales para el crecimiento económico, inclusivo y sostenible, siendo la enseñanza y la formación elementos esenciales para el cumplimiento de dicha agenda (UNESCO, 2016). De aquí la importancia de repensar los procesos formativos de los docentes. Latinoamérica no puede quedarse al margen de esta vorágine de cambios, debe actualizar los programas formativos de los maestros, encaminados a su profesionalización, implementando nuevos modelos que les permita alcanzar la excelencia y calidad en la educación (Castellanos, A., Morga, y Castellanos, N., 2012). 
Es de suma importancia formar y profesionalizar al docente, a partir de un ciclo que inicie con la detección de las carencias y necesidades de aprendizaje, continuando con la oferta de un programa que le permita adquirir las competencias necesarias para su desempeño profesional (Olmedo, y Farerrons, 2014). Al respecto existen varias propuestas de formación, como es el caso del enfoque socioformativo, que se basa en un proyecto ético de vida, el emprendimiento, la colaboración, la co-creación del conocimiento y la metacognición (Tobón, 2017).

Sobre la base de la sistematización de las formas de investigar en ciencias sociales, y particularmente en el ámbito de la educación superior, a partir de la literatura consultada es factible declarar el establecimiento de las siguientes etapas respecto a la manera de proceder en el proceso investigativo educacional, o sea la metodología que se aplique para la investigación está sujeta como regularidad a la siguiente estructura:

Planteamiento del problema: El planteamiento de objetivos, su justificación y viabilidad.

La elaboración del marco teórico: donde se tendrá en cuenta la revisión de la literatura, y propiamente la construcción del marco teórico.

Definir del tipo de investigación: exploratoria, descriptiva o explicativa.

Establecimiento de las hipótesis: definición de las variables.

La selección del diseño de investigación: el diseño experimental o no experimental.

La selección de la muestra: determinación del universo y extracción de la muestra.

Recolección de los datos: que involucra la elaboración de los instrumentos, el cálculo de la validez y aplicación, y el procesamiento de la información.

Análisis de los datos.

Presentación de los resultados: elaboración y presentación de informe que contiene los resultados.

Estos pasos constituyen la lógica científica de la investigación, o sea los principios de demostración e inferencia válida orientada a la obtención de nuevos conocimientos y su aplicación para la solución a problemas o interrogantes de carácter científico.

Como bien se ha analizado la investigación supone, una herramienta necesaria en todos los procesos de crecimiento y desarrollo tecnológico de las empresas, sectores económicos y sociales, no sólo para aquellos de fuerte contenido empresarial, sino para todos los que deben adaptarse a las nuevas exigencias del mundo actual. La universidad en su papel formador de profesionales que ejercen en los diversos sectores reconfigura la investigación.

Ello se aborda desde la doble perspectiva de la investigación básica, como generadora de conocimientos en forma propia, y de la investigación aplicada, donde esos conocimientos son puestos en práctica teniendo en cuenta las intencionalidades de cada sector. En definitiva, la investigación conduce a la modernización de las estructuras productivas y a la integración de las relaciones entre diferentes sectores de la producción y los servicios con la universidad. 


\section{Conclusiones:}

- Es importante la investigación científica porque permite concientizar al profesorado sobre la necesidad de resolución de los problemas reales a los que se enfrenta cotidianamente, permite aplicar la teoría con un carácter creativo e innovador, que pretende encontrar respuesta a problemas trascendentes, a través de la construcción teórica del objeto de investigación y con ello lograr hallazgos significativos que aumentan el conocimiento y lo enriquecen.

- La investigación científica en el contexto de la educación superior provee al investigador de una serie de conceptos, principios y leyes que le permiten encauzar, de un modo eficaz y tendiente a la excelencia el proceso formativo.

- En la concepción de investigación desde la cual se reflexiona, el método científico se fundamenta básicamente en los principios de la dialéctica, donde el fenómeno que se estudia hay que analizarlo objetivamente de forma íntegra y multilateral estando ante todo en la obligación de esclarecer las fuentes internas y la fuerza motriz del desarrollo de los fenómenos y procesos en su evolución, muy vinculado con las características del investigador en su contexto.

- La Metodología de la Investigación Científica estudia las características, las leyes y los métodos de dicho proceso, todo lo cual, en su conjunto, constituye un método teórico de la Investigación Científica como proceso.

\section{Referencias Bibliográficas}

Arias, F. (s.f.). Introducción a la técnica de la investigación en psicología. En F. Arias Galicia, Introducción a la técnica de la investigación en psicología (pág. 28).

Armijos, C. E. G., Barreno, F. L. L., \& Sánchez, F. de J. C. (2015). Los nuevos discursos, normativas y prácticas sobre educación superior en la República del Ecuador: Los énfasis en investigación e innovación. Avanzada Científica, 18 (3), 15-31.

Buckworth, J. (2017). Issues in the Teaching Practicum. En G. Geng, P. Smith, y P. Black (eds.). The Challenge of Teaching (pp. 9-17). (s.l): Springer Singapore. Recuperado de https://researchers.cdu.edu.au/en/publications/issues-in-the-teaching-practicum

Cabero J., y Marín V. (2014). Miradas sobre la formación del profesorado en tecnologías de información y comunicación (TIC). Enlace Revista Venezolana de Información, Tecnología y Conocimiento, 11(2), 11-24. Recuperado de https://goo.gl/YhnwhX

Caliskan, N., Kuzu, O., \& Kuzu, Y. (2017). The Development of a Behavior Patterns Rating Scale for Preservice Teachers. Journal of Education and Learning, 6(1), 130-142. Recuperado de http://www.ccsenet.org/journal/index.php/jel/article/view/64405

Canto, J.L. (2016). Educación en las escuelas Normales: La formación docente en México crítica, tendencia y propuesta. Revista Edução e emancipação, 9(2), 68-85. Recuperado de http://www.redalyc.org/pdf/270/27030105.pdf

Castellanos, N., Morga, L. E., y Castellanos, A. Educación por competencias: hacia la excelencia en la formación superior. México: Red Tercer Milenio. Recuperado de https://goo.gl/koBfMj 
Cid, J. E., Cuadra, L. P., Cuevas, S. H., \& Villalobos, A. A. (2017). Articulación Educación y Trabajo: Un estudio desde la Mirada de los Docentes de la Educación Técnica Regional Chilena y sus Necesidades de Perfeccionamiento Pedagógico. Información tecnológica, 28(1), 25-34.

Díaz, M. F. I. (2017). Perfil diferencial educativo. Análisis de la Realidad Nacional, 114(114).

Espinosa, C. E. F., \& Aguilar, C. E. V. (2017). Desarrollo de habilidades investigativas en la educación superior. Academo, 4 (1), 1-2.

Gadotti, M. (2003). Perspectivas actuales de la educación. Buenos Aires: Siglo XXI.

Hernández, H. y Jiménez, A. (2015). Responsabilidad social empresarial, zona urbana del distrito de Barranquilla: estudio etnográfico. Económicas CUC, 36(2), 9-18. Recuperado de https://revistascientificas.cuc.edu.co/economicascuc/article/view/694/1

Imbernón, F. (2016). Los retos educativos del presente y del futuro. La sociedad cambia, ¿y el profesorado? Revista Internacional de Formação de profesores, 1, 121-129. Recuperado de https://itp.ifsp.edu.br/ojs/index.php/RIFP/article/download/206/373

Maculan, A.-M., Jiménez, C. y Castellanos, O. (2015). Aprendizaje en el proceso de incubación de empresas de base tecnológica. Económicas CUC, 36(1), 15-48

Martínez, M. P. (2014). Proceso de enseñanza-aprendizaje de habilidades sociales y dinámica de grupos en el aula virtual de los ciclos formativos de la Familia Profesional de Servicios Socioculturales a la Comunidad en el régimen semipresencial. Revista Electrónica' Quaderns d' Animació i Educación Social', 19, 115. Recuperado de http://www.emeraldinsight.Com/doi /abs/0.1108 /IJSHE-02-2016-0028

Mas-Torelló, Ó., \& Olmos-Rueda, P. (2016). El profesor universitario en el Espacio Europeo de Educación Superior: la autopercepción de sus competencias docentes actuales y orientaciones para su formación pedagógica. Revista mexicana de investigación educativa, 21(69), 437-470.

Mendoza, D. y López, D. (2015). Pensamiento estratégico: centro neurálgico de la planificación estratégica que transforma la visión en acción. Económicas CUC, 36(1), 8194.

Recuperado

de

https://revistascientificas.cuc.edu.co/economicascuc/article/view/669/26

Mendoza, D., López, D. y Salas, E. (2016). Planificación estratégica de recursos humanos: efectiva forma de identificar necesidades de personal. Económicas CUC, 37(1), 61-79. Recuperado de https://revistascientificas.cuc.edu.co/economicascuc/article/view/1136/pdf_103

Moreno, A. (2015). Enfoques en la formación docente. Ra Ximhai, 11(4), 511-518. Recuperado de http://www.redalyc.org/articulo.oa?id=46142596037

Moriya E. T., Schlünzen, K. J., Nascimento, D. A, (2016). Formación de docentes para el uso de tecnologías digitales. Universal Journal of Educational Research, 4(6), 1288-1297. Recuperado de http://onlinelibrary.wiley.com/doi/10.1111/1471-3802.12138/abstract

Olmedo, N., y Farrerons, O. (2014). Definición, detección, adquisición de competencias y formación de perfiles profesionales en el sector multimedia de las TIC. Revista Internacional de Tecnología, Ciencia y Sociedad, 3 (1), 15-28. Recuperado de http: //journals.epistemopolis.org/index.php/tecnoysoc/article/view/1177

Ortega, P. (2009). Sujetos y prácticas de la pedagogía crítica. Bogotá, D.C.: El Búho.

Padierna, M. P. (2016). La "sociedad del conocimiento" y el campo de las políticas de 
transformación de la educación superior. Revista de educación superior, 45, 117-120.

Recuperado de http://www.redalyc.org/pdf/604/60447470009.pdf

Porlan, R. (1987). El maestro como investigador en el aula. En R. Porlan Ariza, Investigar para conocer, conocer para enseñar (63)

Pozuelo, J. (2014). ¿Y si enseñamos de otra manera? Competencias digitales para el cambio metodológico. Caracciolos, 2 (1), 1-21. Recuperado de https://dialnet.unirioja.Es/ejemplar/377209

Ramírez, R., Chacón, H. y Valencia, K. (2018). Gestión del talento humano como estrategia organizacional en las Pequeñas y Medianas Empresas. Revista CICAG, 16(1): 20-42. Disponible en: http://ojs.urbe.edu/index.php/cicag/article/view/2929

Tejada J., y Ruiz C. (2013). Significación de la práctica en la adquisición de competencias profesionales que permiten la transferencia de conocimiento a ámbitos propios de la acción docente. Revista de Currículum y Formación de Profesorado, 17(3), 91-110. Recuperado de http://www.redalyc.org/pdf/567/56729527006.pdf

Tobón, S. (2017). Conceptual analysis of the socioformation according to the knowledge society. Knowledge Society and Quality of Life (KSQL), 1(1), 9-35. https://goo.gl/aJeSvw

Tedesco, J. C. (1998). Educación y sociedad del conocimiento y de la información. Revista Colombiana de Educación, 36-37, 139-154.

Torres, A. (2007). La educación popular. Trayectoria y actualidad. Bogotá, D.C.: El Búho.

Torres, A. A., y Sánchez, J. J. (2016). Adolescentes y las nuevas formas de relacionarse en la sociedad del conocimiento. TEXTOS. Revista Internacional de Aprendizaje y Cibersociedad, 18(2) ,1-14.Recuperado de http://journals.epistemopolis.org/index.php/textos/article/view/1146

UNESCO. (2016). Estrategia de la UNESCO para la Enseñanza y Formación Técnica y Profesional (EFTP) (2016-2021). UNESCO.

UNESCO. (2017). La educación al servicio de los pueblos y el planeta: creación de futuros sostenibles para todos. Paris: UNESCO.

Zambrano, A. C. (2017). La relación entre conocimiento común y conocimiento científico en el contexto de la enseñanza, aprendizaje y cambio conceptual de las ciencias. TED: Tecné, Episteme y Didaxis, (3).

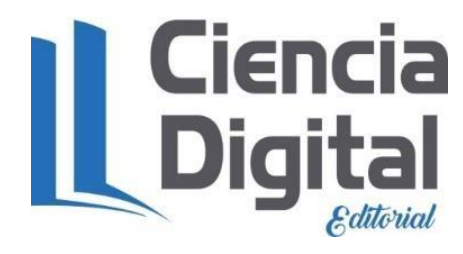




\section{PARA CITAR EL ARTÍCULO INDEXADO.}

Muela Proaño, S. O., Villacis Monar, L. R., Duran Veloz, M. F., \& Tanqueño Colcha, O. P. (2021). Implicaciones valorativas sobre el rol de la investigación científica en el redimensionamiento de la Educación Superior. ConcienciaDigital, 4(1.1), 267-278. https://doi.org/10.33262/concienciadigital.v4i1.1.1558

\section{\Ciencia}

El artículo que se publica es de exclusiva responsabilidad de los autores y no necesariamente reflejan el pensamiento de la Revista Conciencia Digital.

El artículo queda en propiedad de la revista y, por tanto, su publicación parcial y/o total en otro medio tiene que ser autorizado por el director de la Revista Conciencia Digital.

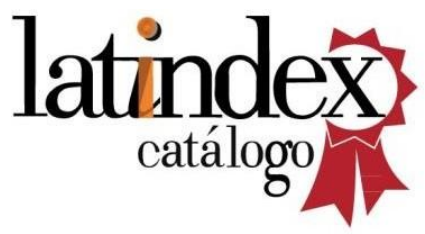

